\title{
A multidrug efflux system is involved in colony growth in Streptomyces lividans
}

Correspondence

Carton W. Chen

cwchen@ym.edu.tw

Received 13 July 2006

Revised 22 November 2006

Accepted 3 January 2007

\author{
Li-Fong Lee, ${ }^{1} \dagger \ddagger$ Yueh-Jung Chen, ${ }^{2} \uparrow$ Ralph Kirby, ${ }^{1}$ Chi Chen ${ }^{1}$ \\ and Carton W. Chen ${ }^{1}$
}

\author{
${ }^{1}$ Department of Life Sciences and Institute of Genome Sciences, National Yang-Ming \\ University, University System of Taiwan, Shih-Pai, Taipei, Taiwan \\ ${ }^{2}$ Department of Biological Science and Technology, Chung Hwa College of Medical \\ Technology, Jen-Te Hsiang, Tainan Hsien, Taiwan
}

\section{INTRODUCTION}

'Multidrug resistance' (MDR) genes are abundant in bacteria; they encode membrane-bound pumps that remove a wide variety of structurally unrelated, lipophilic cationic drugs from the cells. These pumps are actively driven by ATP hydrolysis or by the proton-motive force of the transmembrane proton gradient. Those pumps that are subject to regulatory controls typically belong to the second group and fall into two superfamilies - the major facilitator superfamily (MFS) and the resistance, nodulation and cell division (RND) superfamily (Paulsen et al., 1996; Putman et al., 2000).

tThese authors contributed equally to this work.

Details of the microarray results are available as supplementary data with the online version of this paper.

¥Present address: Biofibre and Biotechnology Application Department, Union Chemical Laboratories, Industrial Technology Research Institute, Hsinchu, Taiwan.

Abbreviations: EB, ethidium bromide; MDR, multidrug resistance; MFS, major facilitator superfamily.
Gram-positive bacteria of the genus Streptomyces are abundant in soil, and must interact extensively with various terrestrial environments. It is thus not surprising that they contain a large number of transporter proteins. For example, there are over 700 predicted transporter genes (about $8 \%$ of all genes) in the Streptomyces coelicolor chromosome (TransportDB, a database for predicted membrane transport protein; http://www.membranetransport.org). At least 100 of the $S$. coelicolor transporters show strong sequence similarity to MDR pumps of various families. Surprisingly, in spite of the large number of MDR pump genes, Streptomyces species are intrinsically sensitive to most of the drugs tested (Lee et al., 1996, 2003). Resistant mutants that arise spontaneously exhibit elevated efflux of the drugs (Lee et al., 1996).

Two classes of spontaneous ethidium bromide (EB)resistant (ebr) mutants have been previously isolated from Streptomyces lividans (Lee et al., 1996). Class I mutants (four members) are resistant to high concentrations of $\mathrm{EB}$ $(>15 \mu \mathrm{M})$, while a class II mutant (one member, YJ18) is resistant to only $5 \mu \mathrm{M}$ EB. The two classes of mutants were mapped at different chromosomal loci and exhibited 
distinct spectra of cross-resistance to other drugs (Lee et al., 2003).

Three EB resistance genes, designated $e b r A, e b r B$ and $e b r C$, were cloned from these mutants based on their ability to confer elevated resistance to EB on a multi-copy plasmid (Lee et al., 2003). Their deduced protein products appear to be transmembrane proteins that resemble other bacterial MDR proteins. EbrA belongs to the small (or staphylococcal) MDR (Smr) family, while EbrB and EbrC belong to the drug resistance translocase family within the MFS. A putative regulatory gene $(e b r R)$ was found downstream of $e b r A$, and another $(e b r S)$ upstream of $e b r C$ in the opposite orientation. Both regulatory genes encode transcription regulatory proteins of the TetR/AcrR family with a putative helix-turn-helix DNA-binding motif, like all other known repressors in this family (Grkovic et al., 2002).

When deletion mutants for each of these genes were analysed, it was found that deletion of $e b r A$ or $e b r B$ did not affect the EB resistance phenotype; hence these genes are not involved in the EB resistance of the ebr mutants (Lee et al., 2003). On the other hand, $e b r C$ appears to be involved in the resistance shown by the class II ebr mutant YJ18, because a disruption of $e b r C$ in YJ18 resulted in the loss of the EB resistance phenotype. YJ18 was found to contain a frameshift mutation in the regulatory gene ebrS that accompanies $e b r C$. Lee et al. (2003) proposed that this mutation caused derepression of $e b r C$ and thus elevated resistance to $\mathrm{EB}$ (and other drugs). ebrC and ebrS are separated by $65 \mathrm{bp}$ and this intergenic region has a relatively high $\mathrm{A}+\mathrm{T}$ content $(43 \mathrm{~mol} \%)$, characteristic of Streptomyces promoter sequences.

The ebrC-ebrS gene pair was physically mapped at about the ' 6 o'clock' position of the S. lividans chromosome (Lee et al., 2003), agreeing with the genetic map location of the ebrS-18 mutation in YJ18 (Lee et al., 1996). Orthologues of the ebrC$e b r S$ gene pair ( $>90 \%$ identity) are present in the same synteny at similar positions on the sequenced chromosomes of S. coelicolor (SCO5516-7) and Streptomyces avermitilis (SAV2727-8). Moreover, ebrC homologues appear to be widespread among Streptomyces species based on a hybridization survey (Lee et al., 2003). All these findings suggest that the ebrC-ebrS pair may play an important biological role.

Many homologues of EbrC with lower degrees of amino acid sequence identity (36-45\%) are also present among uncharacterized proteins (e.g. PqrB) in S. coelicolor (Cho et al., 2003) and S. avermitilis, and among known resistance pumps in other actinomycetes, such as $\mathrm{SgcB}$ of Streptomyces globisporus (Liu \& Shen, 2000), LfrA of Mycobacterium smegmatis (Takiff et al., 1996), RifP of Amycolatopsis mediterranei (August et al., 1998) and VarS of Streptomyces virginiae (Lee et al., 1999).

It is apparent that these MDR systems do not normally confer MDR on their Streptomyces hosts and only derepression of the pump genes achieves elevated resistance. So, what are the biological roles of so many homologues of multidrug efflux pumps in the Streptomyces genomes? In an attempt to shed light on this question, we investigated the genetic control of the ebrS-ebrC regulator-pump pair. We demonstrate here the repressor role of ebrS in regulation of transcription of $e b r C$ and of itself. Several compounds, including rifampicin and $\mathrm{CaCl}_{2}$, were found to induce expression of ebrS. Most notably, deletion of $e b r C$ resulted in retardation of colony growth on certain solid media, and the defect may be suppressed by supplementation of a number of different metal cations. We propose that, under these experimental conditions, the EbrC pump is involved in colony growth through removal of intrinsically produced toxic compounds, and that other MDR systems may also play a similar physiological role in Streptomyces.

\section{METHODS}

Bacterial strains, plasmids and general methods. The bacterial strains and plasmids used in this study are listed in Table 1. Microbiological and genetic manipulations in Escherichia coli and Streptomyces were according to Kieser et al. (2000). Five solid media were used for culturing Streptomyces strains: NE (per litre: $10 \mathrm{~g}$ glucose, $2 \mathrm{~g}$ yeast extract, $2 \mathrm{~g}$ Casamino acids, $1 \mathrm{~g}$ beef extract, $15 \mathrm{~g}$ agar, pH 7.0) (Thiara \& Cundliffe, 1995), PYM (per litre: 5 g peptone, 3 g yeast extract, $3 \mathrm{~g}$ malt extract, $10 \mathrm{~g}$ glucose, $20 \mathrm{~g}$ agar), LB, R5 (Kieser et al., 2000) and HAUCM (Qin et al., 1994).

Dot-blot hybridization. Spores of TK64 or YJ18 were inoculated in $200 \mathrm{ml}$ TSB broth to give an $\mathrm{OD}_{600}$ of about 0.05 , and shaken at $30^{\circ} \mathrm{C}$. Mycelium was collected at different growth phases from early exponential to stationary phases. RNA was isolated by the methods described by Kieser et al. (2000). RNA samples (20-40 $\mu \mathrm{g})$ were applied to a nylon membrane. Hybridization was performed at $45^{\circ} \mathrm{C}$ in a buffer containing $5 \times$ SSPE, $5 \times$ Denhardt's solution, $0.5 \%(\mathrm{w} / \mathrm{v})$ SDS, $50 \%(\mathrm{v} / \mathrm{v})$ formamide and $0.1 \mathrm{mg}$ denatured salmon sperm DNA $\mathrm{ml}^{-1}$. The following probes were labelled with $\left[\alpha{ }^{32} \mathrm{P}\right] \mathrm{dCTP}$ using a random priming kit (Amersham): (i) ebrC: nt 1023-1782; (ii) ebrS: nt 155-774 (GenBank accession no. AY043331); and (iii) 16S rRNA: nt 1-632 (GenBank accession no. X63847).

S1 mapping. Primer PS6 (CTTCTCCAGCAGGCAGCG, in ebrS) and PS7 (AGCATCAGCACGGCGAGC, in $e b r C$ ) were used to prepare by PCR a $0.2 \mathrm{~kb}$ fragment spanning the promoter region, which was used as template for S1 mapping according to Kieser et al. (2000). To map the transcription start site of $e b r C$, PS7 was labelled at the $5^{\prime}$ end with $\left[\gamma^{-32} \mathrm{P}\right] \mathrm{ATP}$ using polynucleotide kinase. The same primer was used for sequence determination in parallel.

Gene disruption. The suicide plasmid pLUS944 was digested with AseI and BglII. The linearized DNA was used to transform protoplasts of TK64 and YJ18 according to Oh \& Chater (1997). After overnight incubation at $30^{\circ} \mathrm{C}$, thiostrepton-resistant transformants were selected by overlaying the agar plates with $200 \mu \mathrm{g}$ thiostrepton $\mathrm{ml}^{-1}$.

Induction of ebrC and ebrS promoters. The basic design of Salah-Bey (1995) was adopted. About $1.5 \times 10^{7}$ spores were spread on a $9 \mathrm{~cm}$ Petri dish containing $25 \mathrm{ml} \mathrm{NE}$ medium supplemented with $30 \mu \mathrm{g}$ kanamycin $\mathrm{ml}^{-1}$. Paper discs $(8 \mathrm{~mm}$; Toyo Roshi) loaded with various test compounds in a volume of less than $20 \mu \mathrm{l}$ were placed on the medium, and the plates were incubated at $30^{\circ} \mathrm{C}$ 
Table 1. Bacterial strains and plasmids

\begin{tabular}{|c|c|c|}
\hline Strain/plasmid & Genotype/description & Source/reference \\
\hline \multicolumn{3}{|l|}{ Bacterial strains } \\
\hline S. lividans 1326 & Wild-type, SLP2 ${ }^{+}$SLP3 $^{+}$ & Hopwood et al. (1983) \\
\hline S. lividans TK64 & pro-2 str-6 SLP2 ${ }^{-} \mathrm{SLP}^{-}$ & Hopwood et al. (1983) \\
\hline S. lividans YJ18 & TK64 containing ebrS-18 mutation & Lee et al. (1996) \\
\hline S. lividans YJ18-8 & YJ18 containing $\Delta(e b r S-e b r C):: t s r$ mutation & This study \\
\hline S. lividans LF13 & TK64 containing $\Delta(e b r S-e b r C):: t s r$ mutation & This study \\
\hline S. lividans LF16 & TK64 containing $\Delta e b r S:$ :tsr mutation & This study \\
\hline S. lividans LF18 & TK64 containing $\Delta e b r S:: t s r$ mutation & This study \\
\hline S. lividans LF24 & TK64 containing $\Delta(e b r S-e b r C):: t s r$ mutation & This study \\
\hline S. coelicolor M145 & $\mathrm{SCP} 1^{-} \mathrm{SCP} 2^{-}$ & Hopwood et al. (1983) \\
\hline \multicolumn{3}{|l|}{ Plasmids } \\
\hline pIJ487 & Streptomyces promoter-probe vector containing tsr and promoterless neo & Ward et al. (1986) \\
\hline pIJ702 & Streptomyces plasmid pIJ101 containing melC and tsr & Katz et al. (1983) \\
\hline pMRC20 & pIJ487 containing the $e b r C$ promoter inserted upstream of neo & This study; Fig. 4A \\
\hline pMCR30 & pIJ487 containing the ebrS promoter inserted upstream of neo & This study; Fig. 4A \\
\hline pLUS914D & $\begin{array}{l}\text { E. coli vector pMTL } 23 \text { containing the } e b r S-e b r C \text { operon with a deletion } \\
\text { of complete } e b r C \text { and most of } e b r S\end{array}$ & Lee et al. (2003) \\
\hline pLUS944 & $\begin{array}{l}\text { E. coli vector pMTL23 containing the ebrS-ebrC operon with a deletion } \\
\text { of most } e b r S\end{array}$ & This study; Fig. 3A \\
\hline pLUS947 & pIJ702 harbouring the $4.0 \mathrm{~kb} S p h \mathrm{I}$ fragment containing wild-type $e b r S$ & This study \\
\hline
\end{tabular}

for 5-10 days. For each drug, $25 \mu \mathrm{g}$ was loaded onto the paper disc. For salts, the following quantities were loaded to each disc: $5 \mu \mathrm{mol}$ $\mathrm{CaCl}_{2}, \mathrm{MgCl}_{2}$ and $\mathrm{MgSO}_{4} ; 0.3 \mu \mathrm{mol} \mathrm{ZnCl}_{2}$; and $0.75 \mathrm{mmol} \mathrm{CuCl}_{2}$.

Microarray analysis. Genomic DNA from a stationary culture was purified by the salting-out procedure (Pospiech \& Neumann, 1995) and sonicated to $<2 \mathrm{~kb}$. Four to six micrograms of sonicated genomic DNA was mixed with $12 \mu \mathrm{g}$ random hexamers (of $72 \mathrm{~mol} \%$ $\mathrm{G}+\mathrm{C}$ content) in a total volume of $25 \mu \mathrm{l}$ and heated at $100^{\circ} \mathrm{C}$ for $10 \mathrm{~min}$. The mixture was quickly cooled on ice before adding the remaining reaction components: $1.5 \mu \mathrm{l}$ Cy3-dCTP or Cy5-dCTP (Amersham Pharmacia Biotech), $4 \mu \mathrm{l}$ Klenow fragment (NEB), $5 \mu \mathrm{l}$ Klenow buffer, $0.5 \mu \mathrm{l}$ dNTP ( $4 \mathrm{mM}$ dATP, $4 \mathrm{mM}$ dTTP, $10 \mathrm{mM}$ dGTP and $0.2 \mathrm{mM} \mathrm{dCTP)}$ and $14 \mu \mathrm{H}_{2} \mathrm{O}$. The random-primed labelling reaction was carried out for $2-3 \mathrm{~h}$ at $37^{\circ} \mathrm{C}$. The reaction mixture was added to $0.5 \mathrm{ml}$ TE buffer $(10 \mathrm{mM}$ Tris/ $\mathrm{HCl}, 1 \mathrm{mM}$ EDTA, pH 8.0) and filtered though a Microcon-30 concentrator (Millipore). The dilution and filtration cycle was repeated twice. The two DNA pools to be compared were mixed in a hybridization mixture containing $3.68 \times$ SSC, $0.18 \%(\mathrm{w} / \mathrm{v})$ SDS and $1 \mu \mathrm{g}$ yeast tRNA (total $16.3 \mu \mathrm{l}$ ), heated at $100{ }^{\circ} \mathrm{C}$ for $5 \mathrm{~min}$ and applied to a UniS $S$. coelicolor PCR array (http://www.surrey.ac.uk/SBMS/Fgenomics/ Microarrays). The two DNA pools to be compared were mixed in a hybridization mixture containing $3.68 \times$ SSC, $0.18 \%(\mathrm{w} / \mathrm{v})$ SDS and $1 \mu \mathrm{g}$ yeast tRNA (total $16.3 \mu \mathrm{l}$ ), heated at $100^{\circ} \mathrm{C}$ for $5 \mathrm{~min}$ and applied to a UniS S. coelicolor PCR array (http://www.surrey.ac.uk/ SBMS/Fgenomics/Microarrays). Hybridization took place under a glass coverslip in a humidified Omnislide (Thermo Hybaid) at $60{ }^{\circ} \mathrm{C}$ for $12-14 \mathrm{~h}$. The slides were washed, dried, and scanned in a GenePix 4000B (Molecular Devices). Mean signal intensity and local background measurements were obtained for each spot on each array by using GenePix Pro 6.0 software, and analysed using Stanford Microarray Database (Gollub et al., 2003). The dataset for each array was normalized using the signals for the S. coelicolor ribosomal protein genes on the array and a mean signal for each gene was calculated. Microarray data were visualized using the program TREEVIEW (Eisen et al., 1998) at a setting of 1.5. In this visualization, red shows hybridization above the dataset norm, green shows hybridization below the dataset norm and black shows mean hybridization close to the norm.

\section{RESULTS}

\section{ebrC is overexpressed in ebr mutant YJ18}

Lee et al. (2003) showed that ebrC is involved in the elevated MDR displayed by the ebr mutant YJ18, presumably because of a frameshift mutation in the adjacent repressor gene, ebrS. To confirm that $e b r C$ was indeed derepressed in YJ18, dotblot hybridization using an ebrC probe was performed to analyse the $e b r C$ transcripts present in YJ18 and its $e b r^{+}$ parent TK64 (Fig. 1). Liquid cultures were collected at different stages of growth, and RNA was extracted for analysis. The results showed that ebrC expression in TK64 in all growth phases was at a very low level that was nevertheless significantly higher than the background level in the control samples (Neurospora crassa and E. coli RNA). In contrast, ebrC expression in YJ18 was highly induced in all growth phases and strongest at stationary phase. Similar analysis using an ebrS probe revealed a very low level of expression of ebrS in both YJ18 and TK64, but nevertheless it was considerably higher in YJ18. These results supported the notion that ebrC is overexpressed in YJ18 because of the defective ebrS. The higher expression of the mutant allele ebrS18 in YJ18 also suggested that ebrS represses its own transcription.

The same conclusions were reached from S1 protection mapping (Fig. 2), which showed increased transcription of 


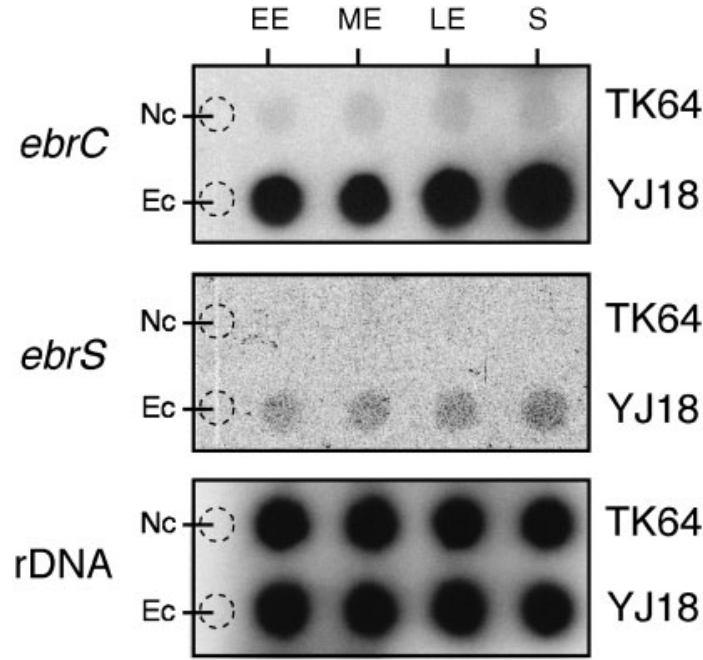

Fig. 1. Expression of ebrC and ebrS in TK64 and YJ18. Total RNA was isolated at four different growth stages of TK64 and YJ18 growing in TSB liquid medium: early exponential (EE; $\mathrm{OD}_{600}$ 0.6-0.7), mid-exponential (ME; $\mathrm{OD}_{600}$ 1.7-1.8), lateexponential (LE; $\mathrm{OD}_{600} 3.9-4.2$ ) and stationary (S; $\mathrm{OD}_{600}$ 1.2-7.8) phases. The RNA was blotted onto a nylon membrane and hybridized with a ${ }^{32} \mathrm{P}$-labelled DNA probe of the ebrC, ebrS and 16S rRNA genes of S. lividans. Neurospora crassa $(\mathrm{Nc})$ and $E$. coli $(\mathrm{Ec})$ RNAs were included as controls. The weak hybridization signals of the ebrS probe were electronically enhanced to reveal the low expression in YJ18.

ebrC in YJ18 in all growth phases from 17 nt upstream of the predicted $e b r C$ coding sequence. A putative -10 region of typical prokaryotic promoters (GACAAT) is found further upstream. The transcription start site for ebrS could not be detected by S1 mapping because of the low expression level.

\section{Disruption of ebrS causes elevated MDR}

To confirm that the elevated ebrC expression in YJ18 was the result of the defect in ebrS, an independent inactivation mutation in ebrS was created in TK64. A suicide vector pLUS944 was constructed that contained a $6 \mathrm{~kb}$ fragment spanning the ebrS-ebrC operon, in which most of the ebrS coding sequence was replaced by the thiostrepton resistance gene (tsr) (Fig. 3A). The vector was used to transform TK64. Thiostrepton-resistant transformants were isolated, in which the wild-type ebrS allele had been replaced by the $\Delta e b r S$ deletion allele on the suicide vector as shown by Southern hybridization analysis (Fig. 3B). Two independent $\Delta e b r S$ transformants (LF16, LF18) of TK64 were isolated on solid medium containing $20 \mu \mathrm{M} \mathrm{EB}$.

Our model also predicted that introduction of a wild-type copy of ebrS on a high-copy-number plasmid would convert YJ18 to a wild-type (EB-sensitive) phenotype. To test this, the $4.0 \mathrm{~kb} S p h \mathrm{I}$ fragment containing the wild-type ebrS gene was inserted into the Streptomyces plasmid vector pIJ702 (Katz et al., 1983). The resultant plasmid, pLUS947, was introduced into YJ18 by transformation. Thiostreptonresistant transformants, YJ18/pLUS947, were isolated and tested for EB sensitivity. The results showed that these transformants had, as expected, become EB sensitive $(3.7 \%$ survival) compared to YJ18 (100\% survival) on $20 \mu \mathrm{M} \mathrm{EB}$.

\section{The ebrS promoter is induced by rifampicin and calcium}

Some bacterial MDR genes are induced by target drugs. For example, in Streptomyces pristinaespiralis, ptr transcription is induced by the substrates pristinamycins I and II, among other unrelated drugs (Salah-Bey et al., 1995). To test the drug inducibility of $e b r C$, a reporter system was constructed. The $0.3 \mathrm{~kb} B g \mathrm{II}-\mathrm{Bam} \mathrm{HI}$ fragment containing the ebrS-ebrC intergenic region was inserted into the BglII site of pIJ487 (Ward et al., 1986), a promoter-probe vector. Two recombinant plasmids were produced with the promoter sequence inserted in opposite orientations (Fig. 4A). In pMRC20, the ebrC promoter was oriented toward the promoterless reporter gene, neo; in pMRC30, the ebrS promoter was oriented toward neo. These two plasmids were introduced into TK64 by transformation. Spores of the transformants were seeded onto a solid medium containing $30 \mu \mathrm{g}$ kanamycin $\mathrm{ml}^{-1}$. Paper discs containing the test drugs were applied to the plates. Induced expression of the neo gene was indicated by the appearance of bacterial growth around the discs (Salah-Bey \& Thompson, 1995).

Eight drugs (rifampicin, chloramphenicol, tetracycline, nalidixic acid, methyltriphenylphosphonium, proflavin, norfloxacin, EB) were tested for induction of ebrC and ebrS expression. The last six drugs are targets of the EbrC efflux system (Lee et al., 1996, 2003). Some salts $\left(\mathrm{CaCl}_{2}\right.$, $\mathrm{MgCl}_{2}, \mathrm{MgSO}_{4}, \mathrm{ZnCl}_{2}$ and $\mathrm{CuCl}_{2}$ ) were also tested.

None of the compounds induced the ebrC promoter in pMRC20 (data not shown). Induction of the ebrS promoter in pMRC30 was exerted by $\mathrm{CaCl}_{2}$ and rifampicin at nonlethal concentrations (Fig. 4B). Interestingly, the induction effects of these two compounds appeared to be synergistic (Fig. 4B, middle panel). Other than these, no other compounds showed any significant induction of the ebrS promoter. In the control experiments, TK64 (Fig. 4B) and TK64/pIJ487 (not shown) failed to grow on the kanamycin plates in the presence or absence of any of the test compounds, ruling out the possibility that the elevated resistance was caused by a physiological effect exerted by the test compounds.

pMRC20 and pMRC30 were also introduced into S. lividans 1326, the ancestor of TK64, and similar results were observed. None of the chemicals tested induced the ebrC promoter, and both $\mathrm{CaCl}_{2}$ and rifampicin induced the ebrS promoter. In addition, $\mathrm{MgCl}_{2}$, which is not an inducer in TK64, induced the ebrS promoter in 1326 (Fig. 4B, bottom panel). The known genetic difference between the two strains is in two plasmids (SLP2 and SLP3 in 1326), proline requirement (pro-2 in TK64) and streptomycin resistance 

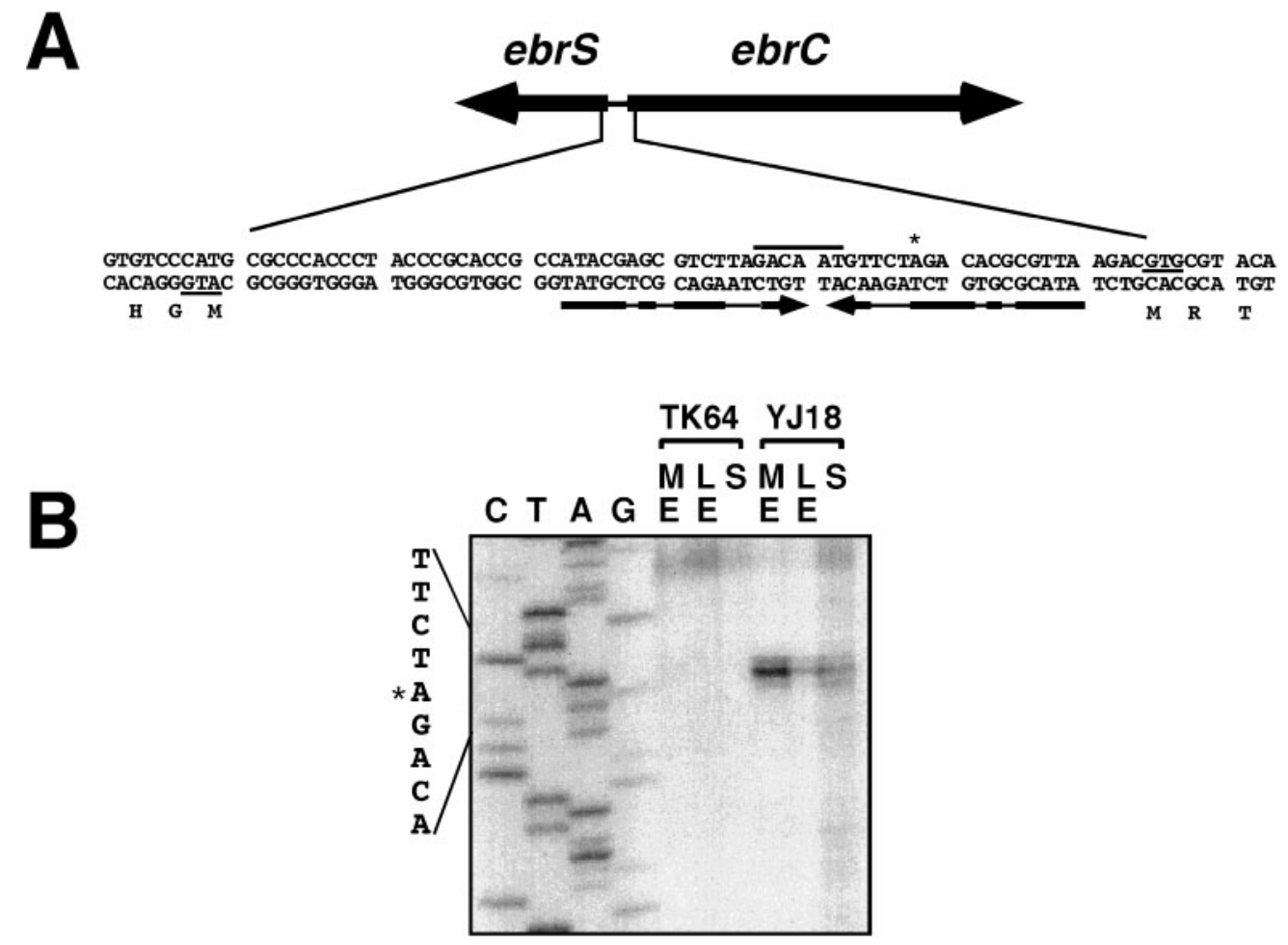

Fig. 2. (A) Promoter region between ebrS and ebrC. The $65 \mathrm{bp}$ intergenic region is flanked by the ebrS and ebrC coding sequences, of which the first three amino acids are shown. The imperfect inverted repeat is underlined by convergent arrows with mismatches shown as thinner lines. The asterisk depicts the transcription start site of ebrC. (B) S1 mapping of the transcription start of ebrC. RNA isolated from TK64 and YJ18 at mid-exponential (ME), late-exponential (LE) and stationary (S; see Fig. 1) phases was subjected to $\mathrm{S} 1$ mapping using two ${ }^{32} \mathrm{P}$-labelled primer sequences in the ebrS and ebrC coding sequences as outlined in Methods. The radioactive signal of the ebrS probe was not detectable and is not shown. The same ebrC primer was used for a sequencing reaction, which is shown on the left-hand side for calibration.

(str-6 in TK64). The reason for this interesting discrepancy is not clear.

\section{The ebrS-ebrC pair is implicated in metal ion metabolism}

The fact that rifampicin and calcium induced the ebrS promoter suggested that they might be involved in the EbrC efflux system. To test this possibility, the $\Delta($ 'ebrS-ebrC) mutation was introduced into TK64 using the ebrS-ebrC suicide vector pLUS914D (Lee et al., 2003) in which the ebrS$e b r C$ sequence was replaced by the $t s r$ gene. Two independent $\Delta(' e b r S$-ebrC) mutants of TK64 (designated LF13 and LF24) were used for further investigation. Similarly, a $\Delta(e b r S-e b r C)$ mutant of YJ18, designated YJ18-8, was created.

All the $\Delta(' e b r S-e b r C)$ mutants exhibited normal colony growth on the isolation medium R5. The presence of rifampicin in the medium reduced both the numbers and sizes of the colonies formed by these mutants (data not shown). YJ18 did not exhibit an increased resistance to rifampicin. Neither did the ebrCS deletion mutants of TK64 (LF13) and YJ18 (YJ18-8) exhibit any increased sensitivity.
These results indicated that rifampicin was not a target of the EbrC efflux system.

$\mathrm{CaCl}_{2}$, on the other hand, had a more complicated effect (Fig. 5A). In the absence of supplemental $\mathrm{CaCl}_{2}$, the colonies of the $\Delta\left({ }^{\prime} e b r S-e b r C\right)$ mutants LF13 and LF24 (not shown) were minute compared to those of TK64 and the $\Delta e b r S$ mutants LF16 and LF18 (not shown) on NE medium. Addition of $\mathrm{CaCl}_{2}$ at $20-300 \mathrm{mM}$ stimulated growth of LF13 and LF24, resulting in a striking increase in colony size with no significant change in colony number. Such growth stimulation was barely detectable in the other four strains. This result indicated that $e b r C$, but not $e b r S$, causes a growth defect on $\mathrm{NE}$ medium that can be suppressed by supplemental $\mathrm{CaCl}_{2}$.

$\mathrm{MgCl}_{2}$ exhibited a similar effect as $\mathrm{CaCl}_{2}$ (Fig. 5B). The poor growth of the LF13 and LF24 colonies was also suppressed by addition of extra $\mathrm{MgCl}_{2}$ up to $150 \mathrm{mM}$. The suppression by $\mathrm{MgCl}_{2}$ appeared to be less effective than that by $\mathrm{CaCl}_{2}$, requiring a concentration of $50 \mathrm{mM}$ for full suppression. $\mathrm{MgSO}_{4}$ exhibited essentially the same suppression effect (data not shown) as $\mathrm{MgCl}_{2}$, indicating that the suppression 
A
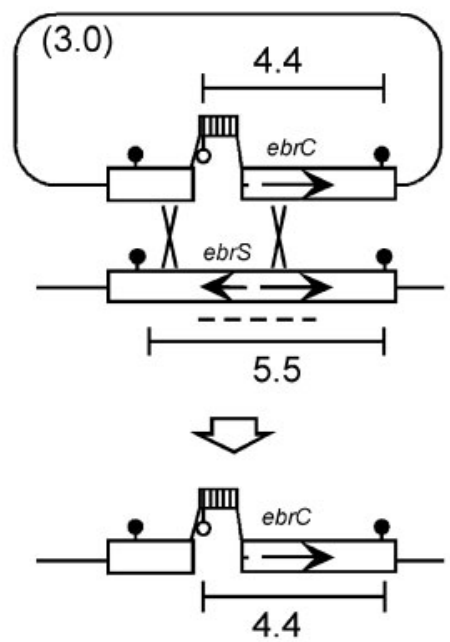

B

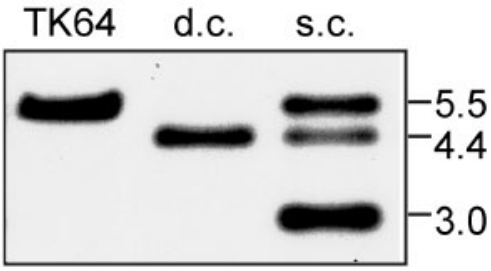

Fig. 3. Insertional inactivation of ebrS. (A) The expected crossovers are shown between the suicide vector pLUS944 and the S. lividans chromosomal sequence. The suicide vector contains a deleted ebrS (replaced by the tsr gene, hatched box) and an intact ebrC gene. The open boxes represent the cloned DNA containing ebrS and ebrC (left and right arrows). The hybridization probes (dashed lines) were the $1.7 \mathrm{~kb} e b r S$ - and ebrCcontaining insert plus the $E$. coli vector plasmid (pUC19 series). The BamHI ( $\bigcirc)$ and Sacl (O) restriction sites, the expected hybridizing restriction fragments and their sizes (in $\mathrm{kb}$ ) are indicated. (B) Confirmation of the replacement in the transformants. Genomic DNA of the transformants was digested with BamHI and Sacl, and subjected to Southern hybridization. d.c., A representative transformant arising by double crossing over; s.c., a representative transformant arising by a single crossover.

was exerted by the cations $\left(\mathrm{Mg}^{2+}\right.$ and $\left.\mathrm{Ca}^{2+}\right)$ and not the anions $\left(\mathrm{Cl}^{-}\right.$or $\left.\mathrm{SO}_{4}^{2-}\right)$.

At high concentrations (500 mM), both $\mathrm{CaCl}_{2}$ and $\mathrm{MgCl}_{2}$ exerted a growth inhibition on all strains, resulting in a reduction in colony size, but insignificant $(<30 \%)$ reduction in colony numbers for all the strains tested. This indicates that there is no apparent involvement of the ebrS$e b r C$ system in resistance to the toxicity of these metal ions.

\section{Specificity of the suppression}

To investigate the specificity of the metal cations, three other divalent cations $\left(\mathrm{Zn}^{2+}, \mathrm{Cu}^{2+}\right.$ and $\left.\mathrm{Mn}^{2+}\right)$ and two
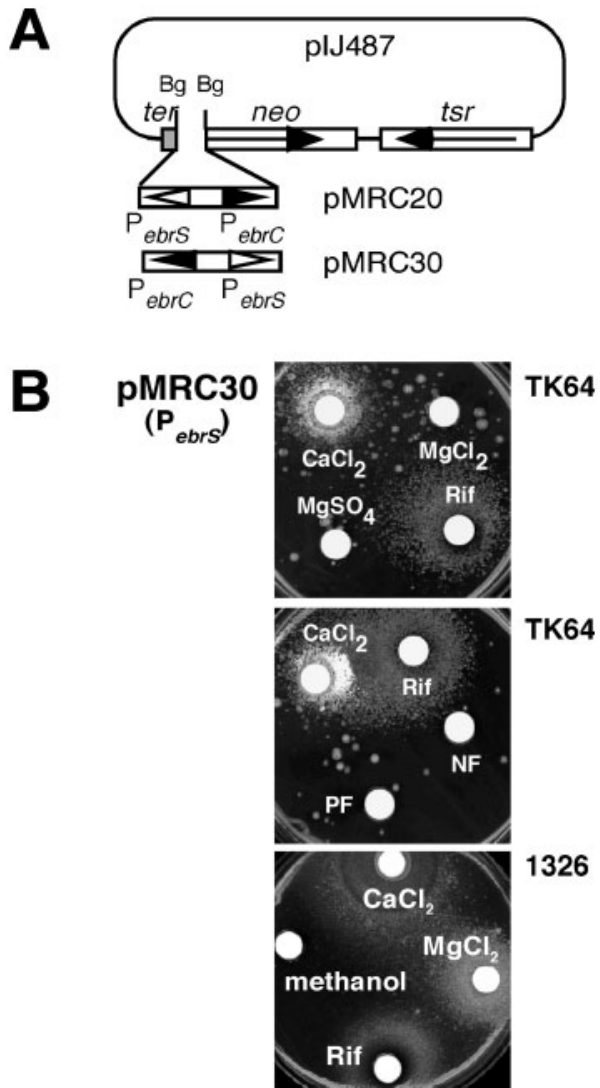

Fig. 4. Induction of the ebrCS promoters. (A) Reporter system for the ebrS and ebrC promoters. The promoter-probe plasmid plJ487 (Ward et al., 1986) harbours an intact tsr gene and a promoterless neo gene (kanamycin resistance). A transcriptional terminator (ter) is located upstream of the neo gene. The $0.3 \mathrm{~kb} \mathrm{Bg} / \mathrm{ll}-\mathrm{BamHI}$ fragment containing the ebrS-ebrC promoter region (boxed arrowheads) was inserted at the $\mathrm{Bg} / \mathrm{ll}$ cloning site $(\mathrm{Bg})$ of plJ487 in two orientations: in pMRC20, the ebrC promoter (filled arrowhead) was oriented toward the promoterless reporter gene, neo; in pMRC30, the ebrS promoter (open arrowhead) was oriented toward neo. pMRC20 and pMCR30 were transferred into S. lividans 1326 (or TK64). (B) Induction of the ebrS and ebrC promoters. Spores of the transformants were spread onto $\mathrm{NE}$ medium containing $30 \mu \mathrm{g}$ kanamycin $\mathrm{ml}^{-1}$. Solutions of each test compound were applied to a paper disc and placed on top of the spread spores. The plates were incubated at $30^{\circ} \mathrm{C}$ for 5 days. Induction of the neo gene resulted in growth of the transformants around the disc. Only results from selected inducers are shown. Rif, rifampicin; NF, norfloxacin; PF, proflavin.

monovalent ions $\left(\mathrm{K}^{+}\right.$and $\mathrm{Na}^{+}$), all as chlorides, were tested for their ability to suppress the $\Delta(e b r S-e b r C)$ mutation effect in LF13 and LF24. Interestingly, none of the divalent ions could suppress the mutation (data not shown), but surprisingly $\mathrm{KCl}$ at $37.5 \mathrm{mM}$ and $\mathrm{NaCl}$ at $100 \mathrm{mM}$ promoted relatively good growth of LF13 and LF24 colonies (Fig. 5C). Thus, the suppression effect is not specific to divalent cations. 

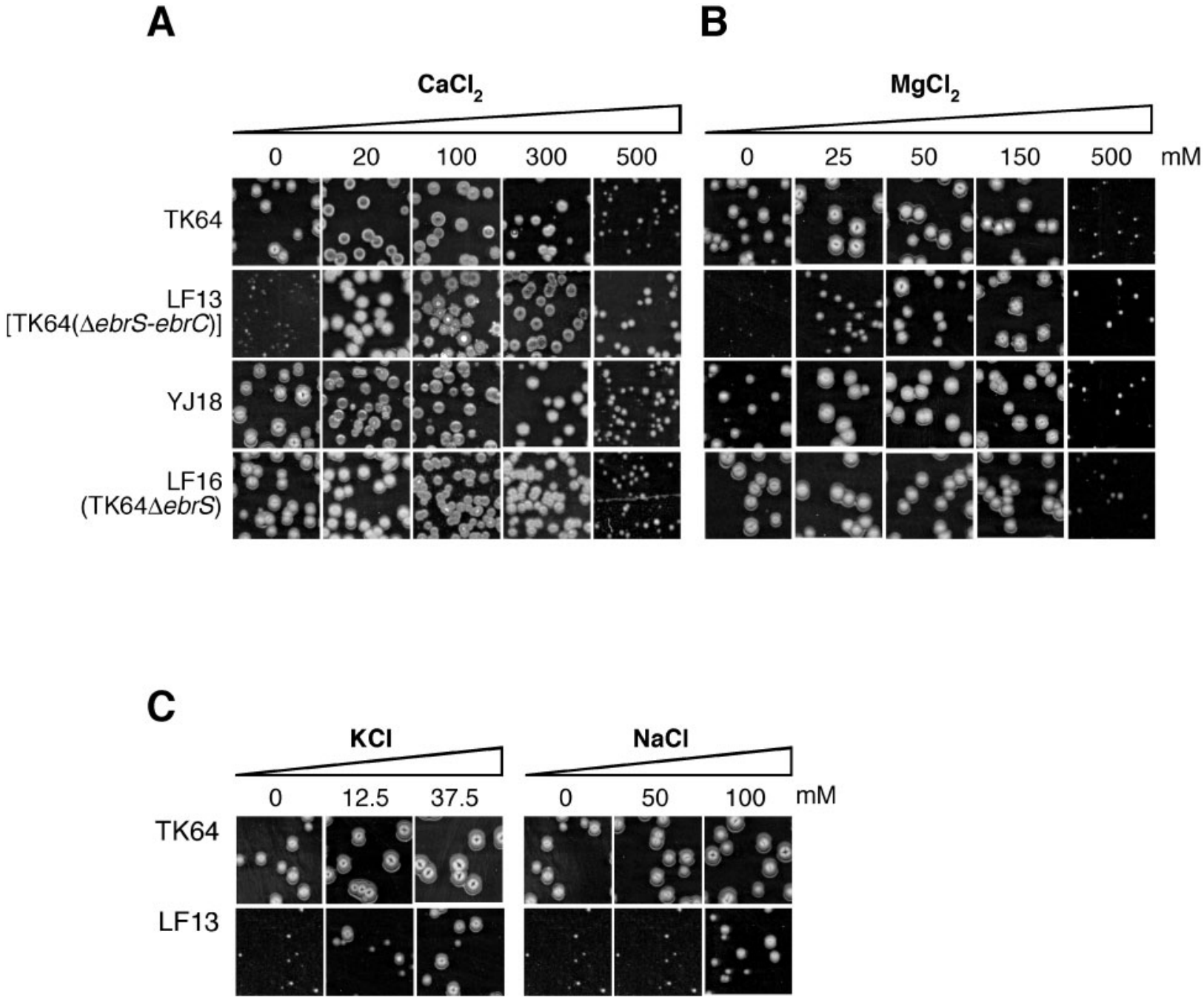

Fig. 5. Colony growth deficiency of the $\Delta(e b r S-e b r C)$ mutants. Diluted spore suspensions were plated on NE medium containing various concentrations of $\mathrm{CaCl}_{2}(\mathrm{~A}), \mathrm{MgCl}_{2}$ (B) and $\mathrm{KCl}$ or $\mathrm{NaCl}(\mathrm{C})$ and incubated for 3 days at $30{ }^{\circ} \mathrm{C}$. The results for LF24 and LF18 were similar to those for LF13 and LF16, respectively, and are not shown. Variations in the colony numbers in each series were insignificant (data not shown).

The growth retardation of LF13 and LF24 under $\mathrm{Ca}^{2+}$ - and $\mathrm{Mg}^{2+}$-limited conditions was also observed on another solid medium, PYM, but not on three other complex media (LB, R5 and HAU). Colonies of the two mutants growing on PYM were retarded to a lesser extent than on NE medium, and supplementation of additional $\mathrm{Mg}^{2+}$ increased the colony size (data not shown).

\section{Genetic instability is not related to the ebr systems}

Streptomyces chromosomes undergo spontaneous deletions of terminal sequences at high frequencies. Some terminally located markers, such as a chloramphenicol-resistance gene (Dyson \& Schrempf, 1987) are lost at frequencies of about $10^{-3}$ to $10^{-2}$ per spore. The reason for this high genetic instability is not known.
This genetic instability is elevated by exposure to DNA intercalating compounds, such as EB (e.g. Crameri et al., 1986; Dyson \& Schrempf, 1987). Therefore, it is possible that the observed 'spontaneous' instability may also be induced by external agents taken up by the cell, some of which may also be substrates for the MDR efflux systems. If so, the ebr mutants with elevated drug efflux might be more genetically stable. Two ebr mutants, YJ18 and YJ51, were compared with the $e b r^{+}$parent TK64 for spontaneous appearance of chloramphenicol-sensitive variants. Spores of YJ18, YJ51 and TK64 were plated on chloramphenicol-free plates and tested for the appearance of chloramphenicolsensitive variants by replica plating. The observed frequencies for the ebr mutants $(0.0-0.1 \%$ for YJ18 and $0.4-0.5 \%$ for YJ51) did not differ significantly from that for the $e b r^{+}$ TK64 (0.1-0.4\%). Therefore, the observed spontaneous genetic instability is not mediated by the target drugs of $e b r C$ 
in YJ18 or an overexpressed unidentified MDR system in YJ51.

\section{Conservation of ebrC and other MDR pump genes among Streptomyces}

Orthologues of $e b r S$ and $e b r C$ (SCO5517 with $100 \%$ amino acid identity and SCO5516 with $99 \%$ amino acid identity) have been previously identified on the chromosome of S. coelicolor (Lee et al., 2003). This pair is also present on the S. avermitilis chromosome (SAV2727 with $61 \%$ amino acid identity and SAV2728 with $81 \%$ amino acid identity) at a similar chromosomal location in the same synteny. A putative orthologue of $e b r C$ ( $36 \%$ amino acid identity), not accompanied by an $e b r S$ homologue, is also found in the unpublished Streptomyces scabies chromosome sequence (http://www.sanger.ac.uk/Projects/S_scabies/; not yet annotated).

To explore the extent of conservation of MDR pump genes, hybridization of genomic DNA from 15 Streptomyces species to 129 of the 133 genes of S. coelicolor classified as multidrug pumps (TransportDB; http://www.membranetransport. org/) on microarrays was analysed (Fig. 6A). The dataset, when calibrated to the mean hybridization signals for the ribosomal proteins, showed that a mean of $89.2 \%$ of the putative MDR genes in S. coelicolor are present in the other Streptomyces species (hybridization signal $\geqslant$ mean -1 $\mathrm{SD}$; dark green to red signals), with the lowest being in Streptomyces clavuligerus (78 \%) and highest in Streptomyces argenteolus $(92 \%)$. The result indicated that most of the

\section{A}

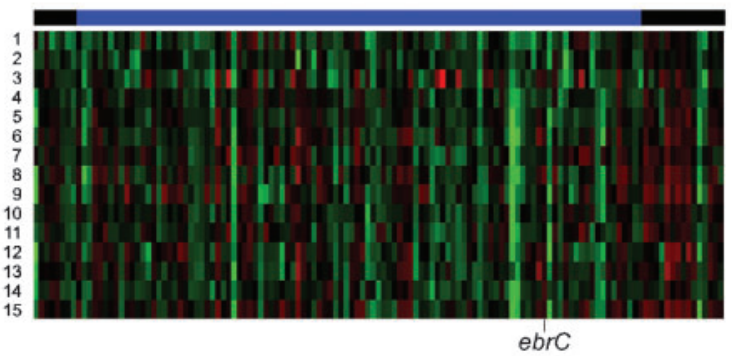

B

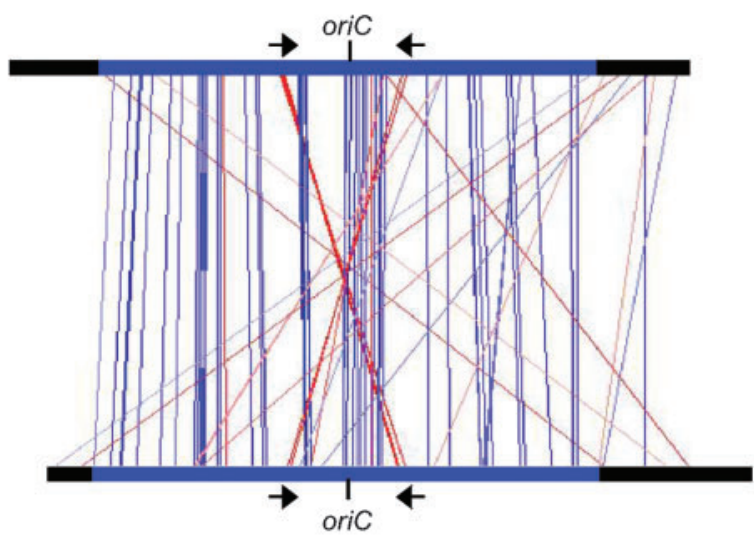

MDR pump genes are conserved among Streptomyces species

Of the 133 designated MDR genes in S. coelicolor, 79 (about $60 \%$ ) found a putative orthologue in S. avermitilis using a maximum expectation value of $10^{-20}$ as criterion. The locations of these orthologue pairs were mapped on the $S$. coelicolor and $S$. avermitilis chromosomes and compared (Fig. 6B). The results showed that about $90 \%$ of the putative MDR genes are located in the highly conserved central region on the two chromosomes (SCO1196-6804 in S. coelicolor and SAV1625-7142 in S. avermitilis) (Ikeda et al., 2003), which contain essentially all the housekeeping genes. These MDR genes display significant conservation in chromosomal locations (parallel connection lines) and orientation ( $86 \%$ of the pairs being in the same orientation; red lines in the inverted regions plus blue lines elsewhere). These results indicate that the majority of the MDR genes in Streptomyces are evolutionarily conserved.

Fig. 6. Conservation of the MDR genes among Streptomyces species. (A) Genomic DNA of 15 Streptomyces species was labelled and hybridized to the UniS S. coelicolor PCR array. Hybridization signals to 129 of the 133 putative MDR pump genes (from TransportDB database) present on the microarray were shown. S. coelicolor M145 genomic DNA was used as the reference sample. The microarray data are represented on a colour scale, with green, black and red representing gene copy numbers less than, equal to or greater than the mean signal level of the ribosomal protein genes. The MDR genes of $S$. coelicolor are arranged in the order from left to right. Streptomyces species: 1, S. clavuligerus ATCC 27064; 2, S. griseus ATCC 10137; 3, S. scabies ISP 5078; 4, S. lipmanii ATCC 27357; 5, S. argenteolus ATCC 23967; 6, S. tanashiensis ATCC 23967; 7, S. hydrogenans ATCC 19631; 8, S. avermitilis ATCC 31267; 9, S. cattleya ATCC 35852; 10, S. venezuelae ATCC 10595; 11, S. rochei 7434-AN4; 12, S. antibioticus IMRU 3720; 13, S. bikiniensis ATCC 11062; 14, S. rimosus ATCC 10970; 15, S. aureofaciens ATCC 10762. The blue horizontal bars mark the highly conserved regions between S. coelicolor and S. avermitilis (Ikeda et al., 2003). Details of the microarray results are available as Supplementary Fig. S1 with the online version of this paper. (B) Syntenic conservation between MDR genes on the $S$. coelicolor and $S$. avermitilis chromosomes. The 133 putative MDR pump genes in $S$. coelicolor were used to search for homologues in the $S$. avermitilis chromosome using TBLASTN (National Center for Biotechnology Information) with a maximum expectation value of $10^{-20}$. The best hit was taken from each of the 93 positive results as an orthologue. Each orthologue pair was mapped to the $S$. coelicolor (upper black bar) and $S$. avermitilis (bottom black bar; flipped to match) chromosomes using the ACT program (Release 2, Sanger Centre), and are connected by blue (same orientation) and red (opposite orientation) lines. An inversion relative to oriC is indicated by the convergent arrows. Seven syntenic orthologous gene pairs are in these regions. The blue horizontal bars mark the highly conserved regions between S. coelicolor and S. avermitilis (lkeda et al., 2003). 


\section{DISCUSSION}

\section{Co-regulation of ebrS and ebrC expression by EbrS}

Divergent transcription of the ebrS-ebrC gene pair from a 65 bp promoter region was found in this study to be coregulated by a common repressor - EbrS. Regulation by a divergently transcribed local repressor gene is common among bacterial MDR systems. The repressors in many cases, like EbrS, belong to the TetR family (reviewed by Grkovic et al., 2002). Mutation in the repressor gene is also a common basis for acquired drug resistance. In Streptomyces, divergently oriented regulator-pump pairs have also been found in antibiotic biosynthesis gene clusters, such as tcmR/tcmA in the tetracenomycin cluster (Guilfoile \& Hutchinson, 1992), actII-orf1/actII-orf2 in the actinorhodin cluster (Fernandez-Moreno et al., 1991), rifP/rifQ in the rifamycin cluster (August et al., 1998) and lanJ/lanK in the landomycin cluster (Westrich et al., 1999). In the pqrAB operon of $S$. coelicolor, mutations in a TetR-type regulatory gene, $p q r A$, result in derepression of the efflux gene, $p q r B$, and elevated resistance to paraquat (Cho et al., 2003). PqrA is also its own repressor. Deletion of the pqrAB operon in wild-type $S$. coelicolor caused supersensitivity to paraquat, indicating that $p q r B$ is expressed at a low level that confers a low level of resistance to paraquat.

\section{Biological significance of calcium- and magnesium-induced ebrS expression}

In this study we showed that the $e b r S$ promoter is induced by high concentrations of $\mathrm{Ca}^{2+}$ in TK64 and by high concentrations of $\mathrm{Ca}^{2+}$ and $\mathrm{Mg}^{2+}$ in 1326. This raises two questions: (i) why do the two strains exhibit different responses to $\mathrm{Mg}^{2+}$; and (ii) why do these ions induce the EbrS repressor and shut down the EbrC pump?

The answer to the first question may only be speculated on, but discrepancy in the induction pattern has also been demonstrated previously in the pristinamycin MDR gene ( $p$ tr) in three Streptomyces species (Salah-Bey et al., 1995). It is notable that the ptr gene is induced by 15 antibiotics (including pristinamycins I and II and rifampicin) and four non-antibiotic chemicals including $\mathrm{CaCl}_{2}$ (Salah-Bey et al., 1995). With respect to the second question, one may rule out $\mathrm{Ca}^{2+}$ itself as the target of the EbrC efflux pump, because the ebrS mutant YJ18 did not exhibit elevated resistance to excessive $\mathrm{Ca}^{2+}$.

\section{Mechanism of calcium and magnesium induction}

In strains TK64 and 1326, $\mathrm{Ca}^{2+}$ may act by binding directly to EbrS and prevent its self-repression. The same goes for $\mathrm{Mg}^{2+}$ in $1326 . \mathrm{Mg}^{2+}$ has been previously shown to complex with tetracycline and the complex binds to the repressor TetR. The binding causes a conformational change in the TetR protein so that it can no longer bind to the tet operator, freeing the tetA promoter for transcription (Hinrichs et al., 1994). Alternatively, $\mathrm{Ca}^{2+}$ and $\mathrm{Mg}^{2+}$ (and probably rifampicin) may act through a common global stress-related adaptive regulon as suggested by Salah-Bey \& Thompson (1995) and Salah-Bey et al. (1995) for ptr gene regulation.

The biological significance of the induction of the ebrS promoter by rifampicin and its synergistic effect with $\mathrm{Ca}^{2+}$ is unclear. Rifampicin is known to increase the copy number of some plasmids, such as ColE1 in E. coli, the replication initiation of which is controlled by RNAs. It is not known whether rifampicin would also affect the copy number of pIJ487 in Streptomyces, which is already high (Kieser et al., 1982). It is noteworthy that rifampicin is not a target of the EbrC efflux system, because the ebrS mutant (YJ18) is no more resistant to rifampicin and $\Delta(e b r S-e b r C)$ mutants (LF13, YJ18-8) are not supersensitive. In contrast, rifampicin is both a target and an inducer for the pristinamycin MDR gene (ptr) of S. pristinaespiralis (Salah-Bey et al., 1995).

\section{Implication of the EbrC pump in waste management}

Despite the presence of multiple MDR genes in their genomes, Streptomyces species, like many other Grampositive bacteria, are sensitive to a multitude of drugs, suggesting that many of the MDR genes are repressed under the tested conditions. It is interesting that none of the five target drugs of EbrC tested in this study showed any induction effect on the expression of EbrC, and the inducing drug, rifampicin, is not a target of the EbrC system. These results are consistent with our previous notion that defence against drugs is probably not the natural biological role of most of the MDR systems in Streptomyces (Lee et al., 2003). It has been postulated that a likely function of these MDR systems is the efflux of undesirable intracellular metabolites (Köhler et al., 1999; Neyfakh, 1997).

Discovery of the growth defect in the $\Delta(e b r S$-ebrC) mutants (but not $\Delta e b r S$ mutants) supports such a postulate. The growth defect of the mutants is attributed to lack of the transporter gene e ebrC and not of the regulator gene ebrS. It is unlikely that the mutants are deficient in any or all of the four suppressing cations, because any of the ions alone is sufficient to restore normal growth. We propose that, under the experimental conditions, the constitutively expressed EbrC pump (Fig. 1) is involved in the efflux of undesirable metabolite products, and that the accumulation of such products retards growth of the mutant colonies on solid medium.

We suggest that many or most of the MDR systems in Streptomyces species are involved in similar intracellular waste treatment tasks. In particular, Streptomyces species and closely related actinomycetes possess large repertoires of gene clusters encoding a wide variety of secondary metabolites in their large genomes. It is likely that more undesirable byproducts are produced in these complex genomes, and it has been beneficial to develop various 
efficient efflux pumps specific for these wastes during evolution. Specificity is important, as it is disadvantageous to remove useful materials from the cells.

MDR genes involved in waste management would be expected to lie in the 'core region' of the Streptomyces chromosomes, which consist mostly of housekeeping genes, whereas the 'arm regions' are dominated by 'conditionally adaptive' genes (Bentley et al., 2002; Ikeda et al., 2003). Of the 79 putative orthologue pairs of MDR pump genes shared by S. coelicolor and S. avermitilis, $90 \%$ are in the conserved Streptomyces-specific core region. These gene pairs are also conserved in chromosomal location and orientation (77\%). Moreover, none of the MDR genes are located in the putative horizontally transferred islands (Bentley et al., 2002). These results support a housekeeping role for the MDR systems.

The waste management model predicts that the many MDR genes are conserved evolutionarily. In this regard, it has been previously shown that homologues of $e b r A, e b r B$ and $e b r C$ appear to be widespread among Streptomyces based on hybridization analysis (Lee et al., 2003). Our microarray and synteny analyses in this study further showed that most of the MDR genes found in S. coelicolor are conserved among other Streptomyces species.

\section{MDR is probably a fortuitous side effect of the waste management systems}

Why then do the MDR pumps display a very relaxed specificity toward extrinsic drugs? We propose that because most of the extrinsic drugs are not the bona fide substrates, they may be exported at very low efficiencies and with low specificity, and overexpressed MDR pumps (through derepression or increased gene copy numbers) are required to achieve sufficient resistance.

In the waste management model, the relevant MDR pumps are expected to be expressed constitutively at a level sufficient for effective removal of undesirable metabolic intermediates (see constitutive expression of ebrC in Fig. 1), and yet insufficient for providing protection against commonly tested drugs (Lee et al., 1996, 2003). In this regard, it is worthwhile to recall that Streptomyces species are generally drug-sensitive, despite the presence of numerous MDR homologues in their genomes (more than 100 in the $S$. coelicolor chromosome). Other bacteria that are relatively susceptible to antibiotics, such as Staphylococcus aureus and Bacillus subtilis, also contain a multitude of MDR genes (Jack et al., 2000; Neyfakh et al., 1991; Ohki \& Murata, 1997). If the role of most MDR systems were the exclusion of extrinsic toxic compounds, the large number of these systems in Streptomyces species would appear to be redundant and unnecessary in view of the wide and relaxed substrate specificity they display.

The mechanism of suppression of the growth defect of the $\Delta(e b r S-e b r C)$ colonies is not clear. It is possible that the added cations exert a physiological effect that reduces the production of the toxic metabolites, and thereby suppresses growth retardation of the $\Delta(e b r S-e b r C)$ colonies. Both $\mathrm{Ca}^{2+}$ and $\mathrm{Mg}^{2+}$ are extracellular signalling cations. As in other bacteria, $\mathrm{Ca}^{2+}$ is expected to be involved in many cellular processes in Streptomyces, and its low cytoplasmic concentration is stringently controlled by dedicated influx and efflux systems (Dominguez, 2004), such as the putative cation transport system encoded by a three-gene cluster (SCO3716-8) in the S. coelicolor genome (Yonekawa et al., 2001). Alternatively, the suppressing cations may act as counter-ions for a transport system that functionally replaces the EbrC pump.

\section{ACKNOWLEDGEMENTS}

We thank David Hopwood for critical comments and suggestion for improvement of the manuscript. This research was supported by research grants from National Science Council, ROC (NSC94-2320-B273-005 to Y.-J. C.; NSC89-2811-B-010-0017 and NSC90-2811-B-010001 to R. K.; NSC93-2312-B-010-004 and NSC94-2321-B010-005 to C.W.C.), and a National Lectureship Award (2001-2004) from Ministry of Education, ROC, to C. W. C.

\section{REFERENCES}

August, P. R., Tang, L., Yoon, Y. J., Ning, S., Muller, R., Yu, T. W., Taylor, M., Hoffmann, D., Kim, C. G. \& other authors (1998). Biosynthesis of the ansamycin antibiotic rifamycin: deductions from the molecular analysis of the rif biosynthetic gene cluster of Amycolatopsis mediterranei S699. Chem Biol 5, 69-79.

Bentley, S. D., Chater, K. F., Cerdeño-Tárraga, A.-M., Challis, G. L., Thomson, N. R., James, K. D., Harris, D. E., Quail, M. A., Kieser, H. \& other authors (2002). Complete genome sequence of the model actinomycete Streptomyces coelicolor A3(2). Nature 417, 141-147.

Cho, Y. H., Kim, E. J., Chung, H. J., Choi, J. H., Chater, K. F., Ahn, B. E., Shin, J. H. \& Roe, J. H. (2003). The pqrAB operon is responsible for paraquat resistance in Streptomyces coelicolor. J Bacteriol 185, 6756-6763.

Crameri, R., Davies, J. E. \& Hütter, R. (1986). Plasmid curing and generation of mutations induced with ethidium bromide in streptomycetes. J Gen Microbiol 132, 819-824.

Dominguez, D. C. (2004). Calcium signalling in bacteria. $\mathrm{Mol}$ Microbiol 54, 291-297.

Dyson, P. \& Schrempf, H. (1987). Genetic instability and DNA amplifications in Streptomyces lividans 66. J Bacteriol 169, 4796-4803.

Eisen, M. B., Spellman, P. T., Brown, P. O. \& Botstein, D. (1998). Cluster analysis and display of genome-wide expression patterns. Proc Natl Acad Sci U S A 95, 14863-14868.

Fernandez-Moreno, M. A., Caballero, J. L., Hopwood, D. A. \& Malpartida, F. (1991). The act cluster contains regulatory and antibiotic export genes, direct targets for translational control by the bldA tRNA gene of Streptomyces. Cell 66, 769-780.

Gollub, J., Ball, C. A., Binkley, G., Demeter, J., Finkelstein, D. B., Hebert, J. M., Hernandez-Boussard, T., Jin, H., Kaloper, M. \& other authors (2003). The Stanford Microarray Database: data access and quality assessment tools. Nucleic Acids Res 31, 94-96. 
Grkovic, S., Brown, M. H. \& Skurray, R. A. (2002). Regulation of bacterial drug export systems. Microbiol Mol Biol Rev 66, 671-701.

Guilfoile, P. G. \& Hutchinson, C. R. (1992). Sequence and transcriptional analysis of the Streptomyces glaucescens tcmAR tetracenomycin C resistance and repressor gene loci. J Bacteriol 174, 3651-3658.

Hinrichs, W., Kisker, C., Duvel, M., Muller, A., Tovar, K., Hillen, W. \& Saenger, W. (1994). Structure of the Tet repressor-tetracycline complex and regulation of antibiotic resistance. Science 264, 418-420.

Hopwood, D. A., Kieser, T., Wright, H. M. \& Bibb, M. J. (1983). Plasmids, recombination and chromosome mapping in Streptomyces lividans 66. J Gen Microbiol 129, 2257-2269.

Ikeda, H., Ishikawa, J., Hanamoto, A., Shinose, M., Kikuchi, H., Shiba, T., Sakaki, Y., Hattori, M. \& Omura, S. (2003). Complete genome sequence and comparative analysis of the industrial microorganism Streptomyces avermitilis. Nat Biotechnol 21, 526-531.

Jack, D. L., Storms, M. L., Tchieu, J. H., Paulsen, I. T. \& Saier, M. H. J. (2000). A broad-specificity multidrug efflux pump requiring a pair of homologous SMR-type proteins. J Bacteriol 182, 2311-2313.

Katz, E., Thompson, C. J. \& Hopwood, D. A. (1983). Cloning and expression of the tyrosinase gene from Streptomyces antibioticus in Streptomyces lividans. J Gen Microbiol 129, 2703-2714.

Kieser, T., Hopwood, D. A., Wright, H. M. \& Thompson, C. J. (1982). pIJ101, a multi-copy broad host-range Streptomyces plasmid: functional analysis and development of DNA cloning vectors. Mol Gen Genet 185, 223-228.

Kieser, T., Bibb, M., Buttner, M. J., Chater, K. F. \& Hopwood, D. A. (2000). Practical Streptomyces Genetics. Norwich: The John Innes Foundation.

Köhler, T., Pechere, J. C. \& Plesiat, P. (1999). Bacterial antibiotic efflux systems of medical importance. Cell Mol Life Sci 56, 771-778.

Lee, L.-F., Huang, Y.-J. \& Chen, C. W. (1996). Two classes of ethidiumbromide-resistant mutants of Streptomyces lividans 66. Microbiology 142, 1041-1047.

Lee, C. K., Kamitani, Y., Nihira, T. \& Yamada, Y. (1999). Identification and in vivo functional analysis of a virginiamycin $S$ resistance gene (varS) from Streptomyces virginiae. J Bacteriol 181, 3293-3297.

Lee, L. F., Huang, Y. J. \& Chen, C. W. (2003). Repressed multidrug resistance genes in Streptomyces lividans. Arch Microbiol 180, 176-184.

Liu, W. \& Shen, B. (2000). Genes for production of the enediyne antitumor antibiotic C-1027 in Streptomyces globisporus are clustered with the cagA gene that encodes the $\mathrm{C}-1027$ apoprotein. Antimicrob Agents Chemother 44, 382-392.

Neyfakh, A. A. (1997). Natural functions of bacterial multidrug transporters. Trends Microbiol 5, 309-313.

Neyfakh, A. A., Bidnenko, V. E. \& Chen, L. B. (1991). Effluxmediated multidrug resistance in Bacillus subtilis: similarities and dissimilarities with the mammalian system. Proc Natl Acad Sci U S A 88, 4781-4785.
Oh, S. H. \& Chater, K. F. (1997). Denaturation of circular or linear DNA facilitates targeted integrative transformation of Streptomyces coelicolor A3(2): possible relevance to other organisms. J Bacteriol 179, $122-127$.

Ohki, R. \& Murata, M. (1997). bmr3, a third multidrug transporter gene of Bacillus subtilis. J Bacteriol 179, 1423-1427.

Paulsen, I. T., Brown, M. H., Littlejohn, T. G., Mitchell, B. A. \& Skurray, R. A. (1996). Multidrug resistance proteins QacA and QacB from Staphylococcus aureus: membrane topology and identification of residues involved in substrate specificity. Proc Natl Acad Sci U S A 93, 3630-3635.

Pospiech, A. \& Neumann, B. (1995). A versatile quick-prep of genomic DNA from gram-positive bacteria. Trends Genet 11, 217-218.

Putman, M., van Veen, H. W. \& Konings, W. N. (2000). Molecular properties of bacterial multidrug transporters. Microbiol Mol Biol Rev 64, 672-693.

Qin, Z., Peng, K., Zhou, X., Liang, R., Zhou, Q., Chen, H., Hopwood, D. A., Kieser, T. \& Deng, Z. (1994). Development of a gene cloning system for Streptomyces hygroscopicus subsp. yingchengensis, a producer of three useful antifungal compounds, by elimination of three barriers to DNA transfer. J Bacteriol 176, 2090-2095.

Salah-Bey, K. \& Thompson, C. J. (1995). Unusual regulatory mechanism for a Streptomyces multidrug resistance gene, ptr, involving three homologous protein-binding sites overlapping the promoter region. Mol Microbiol 17, 1109-1119.

Salah-Bey, K., Blanc, V. \& Thompson, C. J. (1995). Stress-activated expression of a Streptomyces pristinaespiralis multidrug resistance gene ( $p t r)$ in various Streptomyces spp. and Escherichia coli. Mol Microbiol 17, 1001-1012.

Takiff, H. E., Cimino, M., Musso, M. C., Weisbrod, T., Martinez, R., Delgado, M. B., Salazar, L., Bloom, B. R. \& Jacobs, J. W. R. (1996), Efflux pump of the proton antiporter family confers low-level fluoroquinolone resistance in Mycobacterium smegmatis. Proc Natl Acad Sci USA 93, 362-366.

Thiara, A. S. \& Cundliffe, E. (1995). Analysis of two capreomycinresistance determinants from Streptomyces capreolus and characterization of the action of their products. Gene 167, 121-126.

Ward, J. M., Janssen, G. R., Kieser, T., Bibb, M. J., Buttner, M. J. \& Bibb, M. J. (1986). Construction and characterisation of a series of multi-copy promoter-probe plasmid vectors for Streptomyces using the aminoglycoside phosphotransferase gene from Tn5 as indicator. Mol Gen Genet 203, 468-478.

Westrich, L., Domann, S., Faust, B., Bedford, D., Hopwood, D. A. \& Bechthold, A. (1999). Cloning and characterization of a gene cluster from Streptomyces cyanogenus S136 probably involved in landomycin biosynthesis. FEMS Microbiol Lett 170, 381-387.

Yonekawa, T., Ohnishi, Y. \& Horinouchi, S. (2001). A calciumbinding protein with four EF-hand motifs in Streptomyces ambofaciens. Biosci Biotechnol Biochem 65, 156-160.

Edited by: T. Nihira 